\title{
Cytogénétique et cancer
}

Certains remaniements chromosomiques sont spécifiques d'un type de cancer. L'identification des gènes, et notamment des oncogènes situés à proximité des zones remaniées, peut donner de précieuses indications quant au mécanisme du développement tumoral.

\section{Roland Berger \\ Directeur de Recherche au Cnrs. \\ Christian-Jacques Larsen Directeur de Recherche au Cnrs.}

\section{REFERENCES}

I. Varmus $\mathrm{H}$. The molecular genetics of cellular oncogenes. Ann Rev Genet 1984; 18: 553-612.

2. Stéhelin D. Les oncogènes cellulaires, clés de la cancérogénèse. médecine/sciences $1985 ; 1$ : 12-6.

3. Kaplan JC, Szajnert MF. Chromosomes et cancer. Le paradigme du lymphome de Burkitt. médecine/sciences $1985 ; 1$ : 1 7-23.

4. Mitelman F. Catalog of chromosome aberrations in cancer. New York: 2nd ed AR Liss, 1985

5. Yunis JJ, Soreng AL. Constitutive fragile sites and cancer. Science 1984; 226 : 1 199-204.

\section{ADRESSE}

R. Berger, C.-J. Larsen : Institut de recherches sur les maladies du sang, hôpital Saint-Louis, 2, es anomalies chromosomiques des cellules cancéreuses ont été analysées avec les méthodes modernes de la cytogénétique - discipline qui étudie les chromosomes - à partir des années 1960 . Par la suite, l'introduction des techniques de production de "bandes" sur les chromosomes, a permis de décrire un grand nombre d'anomalies chromosomiques des cellules leucémiques et cancéreuses. Parallèlement à ces progrès, des travaux de biologie moléculaire ont mis en valeur l'importance d'une "famille" de gènes, les proto-oncogènes, dans la prolifération maligne [I, 2]. C'est l'emploi conjugué de techniques récentes, telles que l'hybridation in situ sur les chromosomes et le tri des chromosomes à l'aide de la cytofluorométrie de flux, qui rend possible l'étude moléculaire des conséquences des remaniements chromosomiques. $\mathrm{Si}$ le lymphome de Burkitt a été le premier modèle permettant de lier directement remaniement chromosomique et anomalies de segments d'ADN [3], d'autres exemples en sont maintenant connus et leur liste s'accroît à une vitesse impressionnante [4]. Mais l'étude des anomalies chromosomiques des cellules cancéreuses déborde largement le cadre des remaniements dits spécifiques et incite à envisager d'autres aspects biochimiques de la cancérogénèse liés en particulier au dosage génique. La question des sites fragiles [5], qui est encore controversée, ne sera pas envisagée dans cet article.

\section{Anomalies des cellules malignes}

Les anomalies chromosomiques des cellules malignes sont acquises, limitées aux cellules tumorales, et le plus souvent clonales, c'est-à-dire qu'elles dérivent d'une même cellule. On peut distinguer: a) des anomalies de nombre, différent du nombre normal de 46 chromosomes des cellules somatiques normales, aneuploïdie (nombre différent d'un multiple de 23 chez l'homme), polyploïdie (nombre égal à un multiple de 23 supérieur à 2); b) des anomalies dites de structure, définissant des chromosomes " marqueurs" remaniés par rapport à leurs homologues normaux : les plus fréquentes sont les translocations (échange de matériel entre deux chromosomes), les inversions (retournement à I $80^{\circ}$ d'un segment de chromosome), les chromosomes en anneau, des chromosomes dits double minute, de très petite taille, et les segments HSR (homogenous staining regions) colorés de façon homogène avec des techniques de bandes, etc. Ces techniques de bandes dont les méthodes d'obtention sont actuellement variées, permettent d'identifier individuellement avec précision chacun des chromosomes humains et de reconnaître des remaniements 
de structure relativement peu importants. La nomenclature internationale permet d'identifier chaque bande selon une espèce de code. Par exemple le sigle 8q24 désigne la bande 4 de la région 2 du bras long (q) du chromosome 8. Avec les techniques usuelles correspondant à la nomenclature de la Conférence de Paris (I97I) le nombre de bandes est d'environ 350 par génome neutre (22 autosomes + l'X). Une bande " moyenne " correspond donc à environ $1 \times 10^{3}$ kilobases $(\mathrm{kb})$ d'ADN et l'on voit qu'il existe un fossé important entre la résolution de la cytogénétique et celle de la biologie moléculaire qui peut analyser avec les méthodes usuelles jusqu'à roo kb d'ADN. Depuis quelques années, des techniques d'étude des chromosomes dites de haute résolution permettent d'obtenir un plus grand nombre de bandes : 500 à $\mathrm{I} 000$ et peut-être 2000 par génome neutre, ce qui augmente notablement la précision de l'analyse [6]. En outre, le fractionnement de très longs fragments d'ADN, couplé aux techniques dites de "saut " sur les chromosomes [7] accroît notablement les possibilités d'analyse sur de longues distances.

Il est très rapidement apparu que les anomalies chromosomiques n'étaient pas aléatoires. Cependant ce caractère non aléatoire a deux aspects bien différents. Certaines anomalies sont simplement plus fréquentes que ne le voudrait le hasard seul, sans être liées à un type particulier de prolifération maligne. Tel est le cas par exemple de la trisomie 8 (trois chromosomes $n^{\circ} 8$ au lieu de deux) particulièrement fréquente dans les hémopathies malignes, ou les trisomies I partielles des cancers et leucémies. D'autres en revanche, en général des anomalies de structure, sont liées à des types cytologiques particuliers de prolifération. L'exemple le plus connu en est le chromosome Philadelphie $(\mathrm{PhI})$ de la leucémie myéloïde chronique (LMC) qui a été décrit dès 1960.

\section{Anomalies stéréotypées}

Des anomalies chromosomiques stéréotypées, c'est-à-dire identiques d'un cas à l'autre pour une même prolifération, avec les mêmes points de cassure chromosomique sont maintenant bien définis et leur nombre croît rapidement. A la dernière réunion sur la carte génique humaine [8] 85 points de cassures dans 3I néoplasmes ont été rapportés alors qu'il y en avait seulement 44 dans 17 néoplasmes deux ans plus tôt. Quelques-uns des principaux remaniements chromosomiques stéréotypés sont rassemblés dans les tableaux I et II. Les conséquences moléculaires de ces remaniements commencent à être connues. Le tableau II illustre la situation actuelle des tumeurs du système hématopoiétique (leucémies et lymphomes). On peut y constater une coïncidence frappante entre les sites de certains points de cassure et la position de certains proto-oncogènes. De fait, dans plusieurs cas, l'anomalie chromosomique a déplacé le proto-oncogène dans un nouvel environnement, par exemple $c$-myc, dans la forme commune du lymphome de Burkitt portant la translocation $t(8 ; 14)$ ou encore $c$-abl, dans la leucémie myéloïde chronique contenant une translocation $\mathrm{t}(9 ; 22)$ [9], ou bien p53 dans la leucémie promyélocytaire $\mathrm{M}_{3}$ avec translocation $\mathrm{t}(\mathrm{I} 5 ;$ i 7$)$ [Io]. Mais dans d'autres cas, l'anomalie peut laisser le proto-oncogène à son site normal et juxtaposer à proximité un segment d'ADN susceptible de perturber l'expression du gène (c'est le cas des formes variantes du lymphome de Burkitt où $c$-myc est juxtaposé à un segment d'un gène d'immunoglobuline) [3]. Les cas négatifs où l'on n'a observé ni déplacement de proto-oncogène, ni remaniement moléculaire dans un locus ou à proximité de ce locus ne contredisent pas les résultats précédents car les techniques courantes ne permettent pas d'explorer l'ADN d'une bande chromosomique sur des distances suffisamment grandes. A côté des proto-oncogènes, il est clair que d'autres gènes peuvent être altérés par les remaniements chromosomiques. Ce fait a amené certains groupes à leur attribuer un statut de proto-oncogène, car leur activation les rendrait aptes à participer au processus de transformation maligne. Ainsi, les translocations $\mathrm{t}(\mathrm{II} ; \mathrm{I} 4)$ et $\mathrm{t}(\mathrm{I} 4 ; \mathrm{I} 8)$ identi- fiées dans les lymphomes de type $B$ ont pu activer deux gènes bcli [ [ I I] et bcl2 [12, I3] en les juxtaposant aux gènes de la chaîne lourde d'immunoglobuline. Selon Tsujimoto et coll. [12, I4], la translocation aurait pour origine un appariement illégitime de séquences homologues " 7 mères-9 mères ", qui assurent normalement les réarrangements somatiques des gènes d'immunoglobulines des lymphocytes B ( $f$ gure $I$ ). S'il en est ainsi, il est logique de retrouver le même mécanisme de recombinaison à l'origine des translocations du lymphome de Burkitt, puisqu'un gène d'immunoglobuline est impliqué dans les trois formes cytogénétiques de la maladie. Par ailleurs, ce mécanisme est compatible avec la position permanente de $c$-myc en $5^{\prime}$ d'une région constante $(\mathrm{C})$ du gène d'immunoglobuline.

Les anomalies chromosomiques non aléatoires les plus fréquemment retrouvées dans des leucémies et lymphomes de type $\mathrm{T}$ affectent significativement la bande qI I du chromosome I4 où est localisé le gène de l'une des sous-unités du récepteur T (sous-unité $\alpha,\left[I_{5}, \mathrm{I} 6\right]$ ). Comme les gènes d'immunoglobuline, les gènes codant pour les sousunités du récepteur $T$ subissent des réarrangements somatiques au cours de l'activation des cellules $T$, vraisemblablement par des mécanismes similaires de recombinaison. Il est intéressant que dans deux cas de lignées établies à partir de leucémies $T$ et dans des cellules fraîches avec translocation $t$ (8; I4) (q24; q I I ), c-myc soit placé dans le voisinage immédiat du gène du récepteur $\mathrm{T}[\mathrm{I} 7, \mathrm{I} 8]$. On retrouve donc dans les cellules $\mathrm{T}$ une situation symétrique à celle que l'on rencontre dans le lymphome de Burkitt.

Pour illustrer les conséquences de ces remaniements, nous n'examinerons pas ici le cas du lymphome de Burkitt [3] mais celui du chromosome Philadelphie de la LMC. On sait que le chromosome Philadelphie correspond habituellement à une translocation $\mathrm{t}(9 ; 22)$ ( $\mathrm{q34} ; \mathrm{q}$ I I ) et qu'il peut être observé dans certaines leucémies aiguës myéloblastiques (LAM) et lymphoblastiques (LAL). Mais il existe des translocations variantes, soit à trois chromo- 


\section{REFEERENCES}

6. ISCN (1985): An International System for Human Cytogenetic Nomenclature, Harnden DG, Klinger HP, eds. Published in collaboration with Cytogenet Cell Genet. Basel : Karger, 1985.

7. Collins FS, Weissman SM. Directional cloning of DNA fragments at a large distance from an initial probe; a circularization method. Proc Natl Acad Sci USA 1984; 81 : 6812-6.

8. Berger R, Bloomfield CD, Sutherland GR. Report of the committee on chromosome rearrangements in neoplasia and on fragile sites. Human Gene Mapping 8. Cytogenet Cell Genet 1985; 40 : 490-535

9. De Klein A, Geurts van Kessel A, Grosveld $G$, et al. A cellular oncogene is translocated to the Philadelphia chromosome in chronic myelocytic leukaemia. Nature 1982; 300: 765-7.

Io. Le Beau MM, Westbrook CA, Diaz MO Rowley JD, Oren M. Translocation of the P53 gene in $t(15 ; 17)$ in acute promyelocytic leukaemia. Nature 1985; 316: 826-8.

I1. Erikson J, Finan J, Tsujimoto Y, Nowell PC, Croce CM. The chromosome 14 breakpoint in neoplastic B cells with the $t(11 ; 14)$ translocation involves the immunoglobulin heavy chain locus. Proc Natl Acad Sci USA 1984; 81 : 4144-8.

12. Tsujimoto Y, Gorham J, Cossman J, Jaffe E Croce CM. The t $(14 ; 18)$ chromosome translocations involved in B-cell neoplasms result from mistakes in VDJ joining. Science 1985; 229: 1390-3.

13. Bakhshi A, Jensen JP, Goldman P, et al. Cloning the chromosomal breakpoint of $t(14 ; 18)$ human lymphomas: Clustering around $\mathrm{J}_{H}$ on chromosome 14 and near a transcriptional unit on 18. Cell 1985; 4 1 : 899-906.

14. Tsujimoto Y, Jaffe E, Cossman J, Gorham J, Nowell PC, Croce CM. Clustering of breakpoints on chromosome 11 in human B-cell neoplasms with the $t(11 ; 14)$ translocation. Nature 1985; $315: 340-3$.

15. Lewis WH, Michalopoulos EE, Williams DL, Minden MD, Mak TW. Breakpoints in the human $\mathrm{T}$-cell antigen receptor $\alpha$-chain locus in two T-cell leukacmia pat:ents with chromosomal translocations. Nature 1985; 317 : 544-6.

16. Erikson J, Williams DL, Finan J, Nowell PC Croce CM. Locus of the $\alpha$-chain of the T-cell rereptor is split by chromosome translocation in T-cell leukacmias. Science $1985 ; 229$ : 784-6.

17. Mathieu-Mahul D, Caubet jF, Bernheim A et al. Molecular cloning of a DNA fragment from human chromosome 14 (14911) involved in T-cell malignancies. Embo 7 1985; 4 : 3427-33.

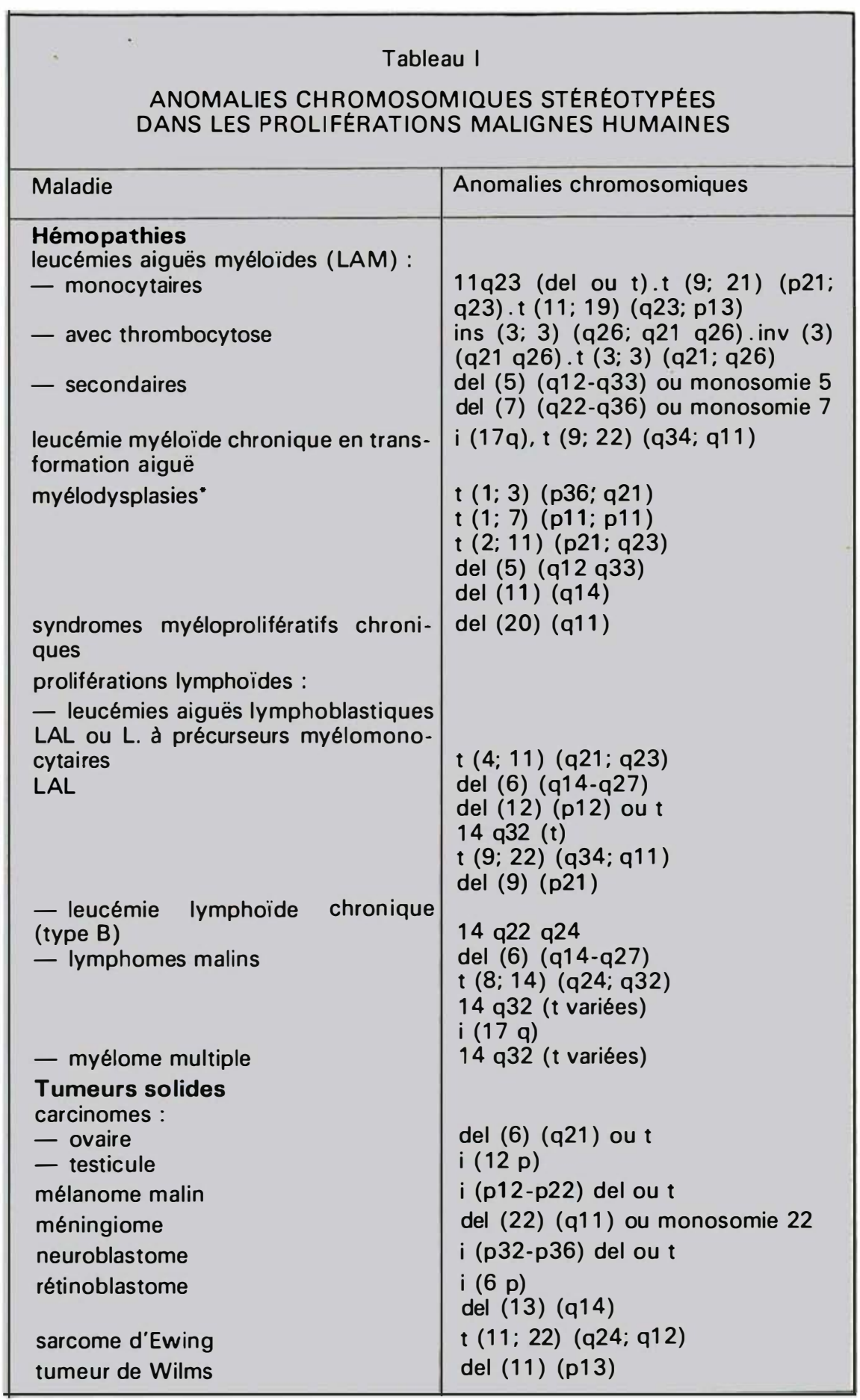

Seules sont indiquées ici les anomalies de structure définitivement établies [8] del : délétion, $\mathrm{i}$ : isochromosome, ins : insertion, inv : inversion, $\mathrm{t}$ : translocation. - Anomalies pouvant être également observées dans des LAM et des syndromes myéloprolifératifs chroniques.

somes, impliquant un $\mathrm{n}^{\circ} 9$ et un $\mathrm{n}^{\circ} 22$, soit à deux chromosomes impliquant le 22 et un autre chromosome. Du fait de la translocation $t$ (9; 22) la séquence $c$-abl normalement localisée sur la bande 9934 , est transférée sur le chromosome 22 remanié. Il en est de même dans les translocations variantes, y compris celles où le remaniement du chro- 
mosome 9 n'est pas évident cytogénétiquement, ce qui montre l'unicité du chromosome $\mathrm{Ph}$.

Les travaux remarquables de l'équipe de Rotterdam ont démontré que la translocation juxtapose $c$-abl et une séquence d'ADN du chromosome 22 [I9]. La très grande majorité des points de cassure sur le chromosome 22 est localisée dans cette séquence d'environ 6 kilobases qui a reçu le nom de breakpoint cluster region ou bcr. On sait désormais que le gène $c$-abl est exprimé sous la forme d'un ARNm chimérique contenant des séquences bcr et $c-a b l[20]$. Cet ARN (plus grand que l' ARNm normal) est traduit en une protéine hybride de 2rokd qui possède une activité tyrosine kinase dont est dépourvue, dans les conditions du test, la protéine $c-a b l$ normale qui est plus légère ( $140 \mathrm{kd})$ [2I].

\section{Anomalies non spécifiques}

A côté des anomalies chromosomiques stéréotypées, et parfois associées à elles, il existe toute une série d'anomalies non aléatoires et non spécifiques d'un type cytologique donné de prolifération. Certaines de ces anomalies sont considérées comme secondaires par rapport aux remaniements spécifiques. En effet,

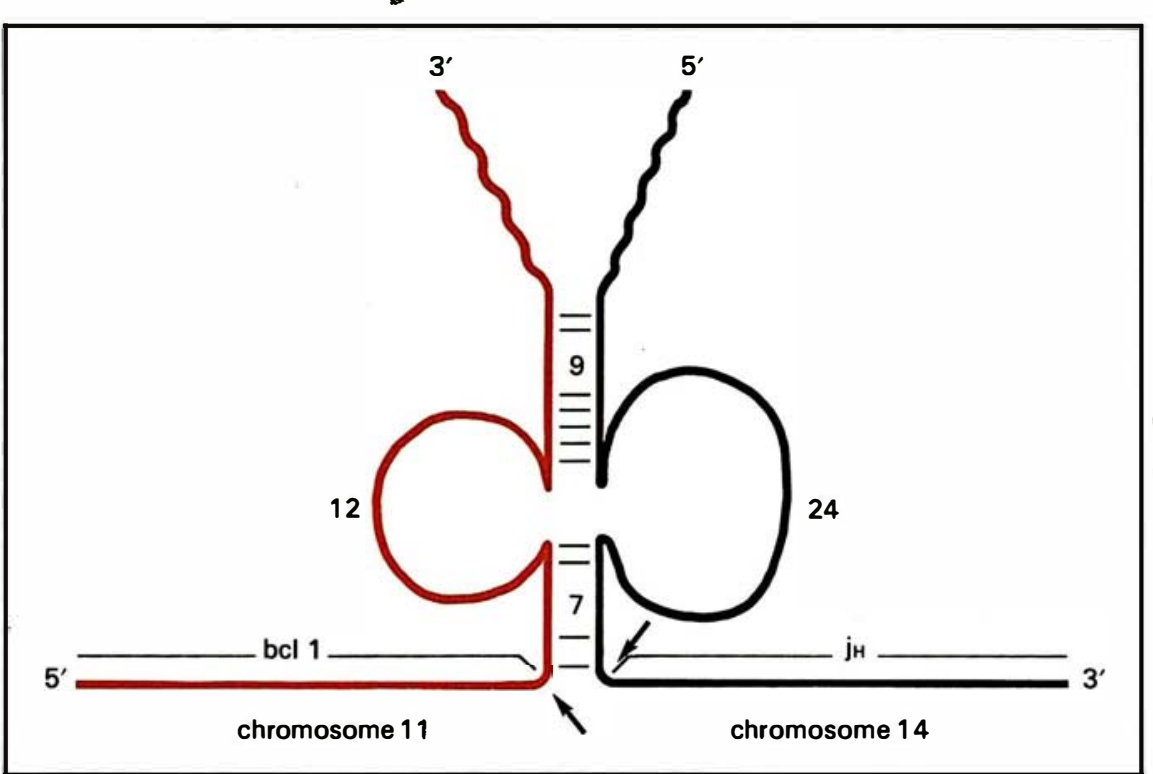

Figure 1. Schéma explicatif du mécanisme de jonction entre les chromosomes 11 et 14 dans une leucémie de type $B$ avec translocation $t$ (11; 14) (q13; q32). (Selon Tsujimoto et al.). Dans le gène de la chaíne lourde d'immunoglobuline $(\mathrm{lgH})$, les régions $D_{H}$ et $\mathrm{J}_{H}$ sont flanquées respectivement en $3^{\prime}$ et $5^{\prime} d e$ structures heptamère-nonomère (" 7 mères-9mères ॥) séparées par une séquence de 12 ou 24 nucléotides (spacer). Au cours du réarrangement somatique du gène de I'lgH, la structure 7 mères-9mères de l'une des régions $D_{H} s^{\prime}$ apparie avec une structure 7 mères -9 mères d'une région $J_{H}$. Une enzyme spécifique ou recombinase clive les 2 brins (au niveau des flèches sur le schéma) et place les régions $D_{H}$ et $J_{H}$ concernées, en continuité.

L'analyse de la séquence nucléotidique en 3 ' de la région bcl 1 sur le chromosome 11 a montré la présence d'un 7 mères-9 mères. Un appariement illégitime entre deux chromosomes différents, suivi d'une scission-ligature catalysée par la recombinase, serait à l'origine de la translocation observée. Dans l'exemple cité, bcl1 se substitue à la région $D_{H}$. A noter que la complémentarité n'est pas parfaite au niveau des séquences 7 mères 9 mères. A noter également que ce mécanisme ne semble pas commun à tous les lymphomes $B$ porteurs de translocation $t(11 ; 14)$. Par contre, un mécanisme similaire existe pour la translocation $t(14 ; 18)$ du lymphome folliculaire à cellules $B$. lorsque ces deux types d'anomalies coexistent dans un même clone cellulaire, les anomalies secondaires peuvent varier d'un cas à l'autre. Un autre argument réside dans le fait que des anomalies secondaires peuvent apparaître au cours de l'évolution des tumeurs ou des leucémies, ce qui définit l'évolution clonale du caryotype. L'exemple le mieux étudié d'évolution clonale est celui de la transformation aiguë de la LMC dans laquelle des anomalies chromosomiques clairement non aléatoires se surajoutent au chromosome $\mathrm{Ph}$. Ces anomalies secondaires peuvent être structurales, comme le sont la plupart des anomalies primitives, ou numériques. Elles impliquent alors soit des trisomies ou des polysomies correspondant à une amplification génique et donc à un déséquilibre quantitatif cellulaire, soit à des pertes de chromosomes, permettant à d'éventuelles mutations récessives de s'exprimer au niveau de la cellule, puisque l'allèle correspondant supposé non muté est perdu.

L'étude précise des remaniements chromosomiques des néoplasies est rendue souvent difficile par leur complexité même. Elle est cependant importante pour comprendre quelle est la part des anomalies primitives et secondaires, et quelles sont les conséquences moléculaires attendues. Du fait de la juxtaposition de nombreuses anomalies, l'analyse des anomalies chromosomiques devrait aider à préciser la question de la coopération entre oncogènes, qui apparaît d'une importance considérable dans les conditions pathologiques.

En effet, une anomalie primaire peut activer un proto-oncogène et des anomalies surajoutées, " secondaires", activant à leur tour d'autres gènes, pourront faciliter l'émergence de sous-clones tumoraux à grandes capacités de prolifération et de dissémination.

\section{Amplification}

Les chromosomes double minute (dm) sont des éléments de petite taille (correspondant à $0,3-0,5 \mu \mathrm{m}$ ) groupés par paires et en nombre variable selon les cellules, mais parfois très nombreux. Des travaux 


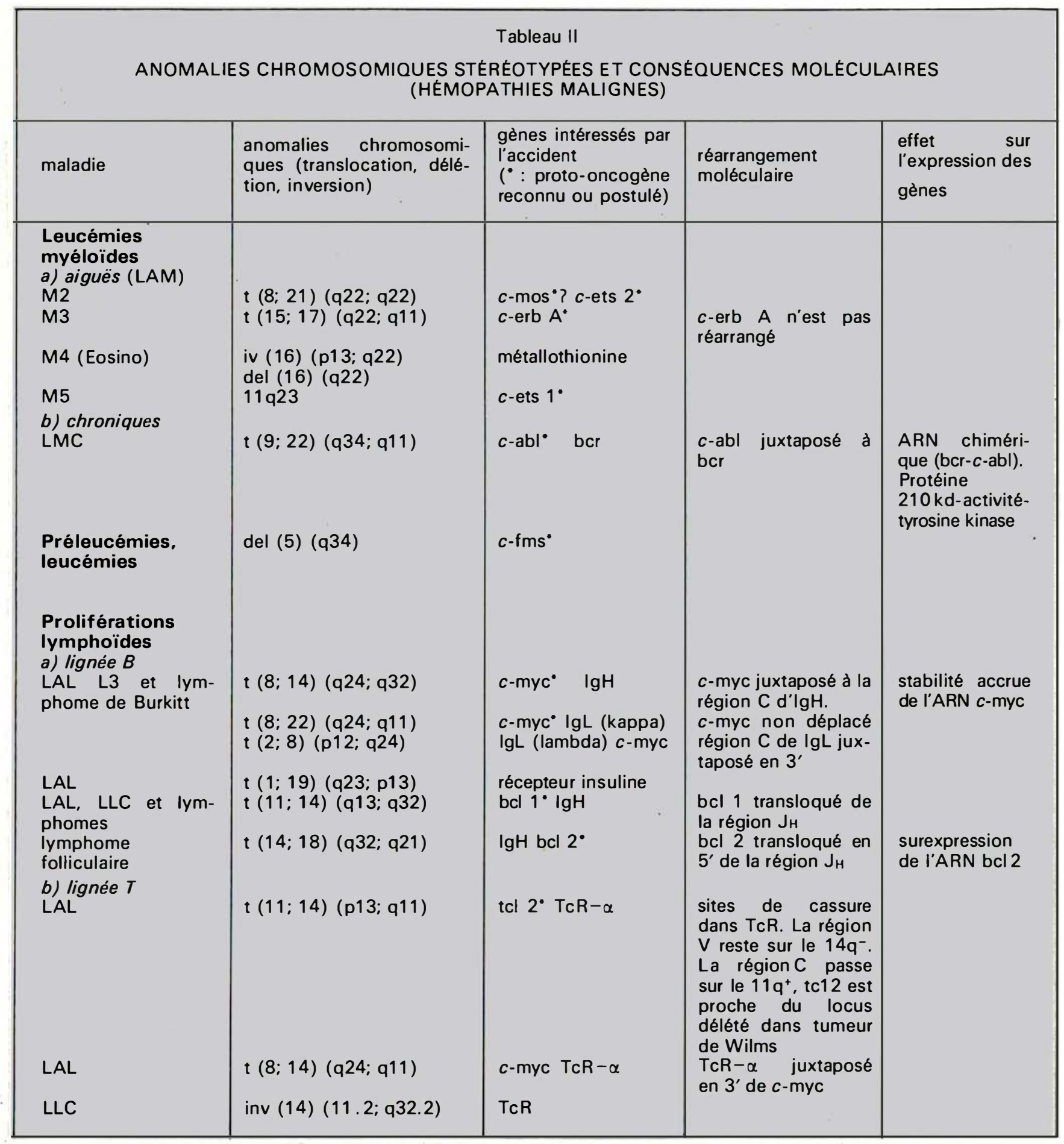

LMC : Leucémie myéloïde chronique. LAL : Leucémie aiguë /ymphoblastique. LLC : Leucémie lymphoïde chronique.

expérimentaux [22] ont montré qu'ils étaient équivalents aux segments dits HSR car selon les conditions de culture in vitro ou in vivo, le passage entre ces deux formes cytologiques est possible. Les chromosomes $\mathrm{dm}$ sont particulièrement fréquents dans certaines tumeurs comme les neuroblastomes, mais ils peuvent être observés, comme les HSR, dans n'importe quel type de tumeur maligne, plus 
fréquemment cependant dans les tumeurs solides que dans les hémopathies. Leur étude a été facilitée par leur présence dans des lignées cellulaires établies à partir de tumeurs ou de leucémies spontanées ou expérimentales. On sait maintenant que HSR et dm sont le support d'une amplification génique, qui peut varier de quelques dizaines à plusieurs dizaines de milliers de séquences d'ADN. A ce jour, deux types de gènes ont été identifiés dans les séquences amplifiées : gènes de résistance à des agents chimiques utilisés en thérapeutique (méthotrexate, correspondant au gène codant pour la dihydrofolate réductase, gènes de polyrésistance aux antitumoraux) et oncogènes cellulaires en particulier $c$-myc dans des lignées leucémiques ou de cancer du colon, $\mathrm{N}$-myc dans des neuroblastomes, $c$-myc et $\mathrm{N}$-myc dans des cancers du poumon, gènes de la famille ras dans des tumeurs variées [23]. La localisation des HSR dans le caryotype ne correspond en général pas à celle du gène normal et il existe une certaine instabilité dans la localisation chromosomique des HSR. De plus, il apparaît que dans certains cas, en particulier dans celui des neuroblastomes, le nombre de $\mathrm{dm}$ ou leur taille, ou le nombre de HSR augmente au cours de l'évolution de la tumeur. Cet accroissement de l'amplification génique pour les oncogènes pourrait donc en pratique servir à évaluer le degré d'évolution des tumeurs [24]. La signification précise de ces amplifications géniques est encore l'objet de spéculations. Dans la plupart des cas l'amplification des gènes de résistance paraît clairement liée à l'emploi d'agents chimiques et est donc d'acquisition secondaire; dans d'autres cas, l'amplification semble précéder leur utilisation. De façon similaire, l'observation d'une amplification d'oncogènes au moment du diagnostic n'autorise pas à considérer qu'elle est un événement primaire puisque la tumeur a déjà évolué avant d'être reconnue. Quoi qu'il en soit, l'amplification paraît conférer un avantage sélectif aux cellules qui la portent et on admet qu'elle correspond à un des mécanismes d'activation des oncogènes cellulaires, puis- qu'elle induit une surexpression du gène amplifié. Une question essentielle est de savoir quelle est l'organisation moléculaire des gènes amplifiés. Les séquences d'ADN amplifiées sont constituées par la succession colinéaire de copies du gène. Dans certaines tumeurs, les séquences paraissent intactes au regard des cartes de restriction; dans d'autres, elles paraissent avoir subi de nombreux remaniements montrant que les mécanismes moléculaires d'amplification correspondent à des remaniements géniques complexes et certainement multiples.

\section{Homozygotie et hémizygotie}

L'homozygotie correspond au fait que les deux allèles d'un gène donné sont identiques sur les deux chromosomes homologues, et l'hémizygotie au fait qu'il n'y a qu'un seul gène par cellule au lieu de deux, l'un d'entre eux ayant été perdu.

Le rétinoblastome est une tumeur maligne de la rétine qui peut être transmisé génétiquement sur le mode dominant. Les cytogénéticiens ont montré que dans une certaine proportion de cas (quelques pour cent) les sujets porteurs de rétinoblastome ont une délétion qui peut être minime et correspondre à une seule bande sur le chromosome I3 au niveau de la bande I 3 qI 4. Cette anomalie est constitutionnelle, ce qui signifie qu'elle existe dans toutes cellules de l'organisme, et elle est à l'état hétérozygote, c'est-à-dire qu'il existe chez ces sujets un chromosome I3 porteur de la délétion et un chromosome I 3 normal [25]. Dans les cellules tumorales elles-mêmes il peut exister de nombreuses anomalies chromosomiques, et en particulier des anomalies impliquant les chromosomes 13 : il s'agit alors le plus souvent de la perte du chromosome I3 normal qui peut être remplacé par le chromosome I3 délété (homozygote pour le I 3 anormal) ou non (hémizygote)[26]. Tout se passe donc dans ces cas comme si le chromosome i 3 normal compensait la délétion de son homologue (d'où le terme d'anti-oncogène pour désigner les séquences d'ADN correspondantes). Quel que soit le mécanisme aboutissant à l'homozygotie du chromosome I3, anomalies de ségrégation mitotique, crossing-over somatique, l'existence du phénomène est en accord avec les modèles à plusieurs étapes de cancérogénèse : la délétion constitutionnelle serait la première étape, l'homo- ou l'hémizygotie, une des étapes ultérieures. L'utilisation de marqueurs géniques ou de fragments de polymorphisme de restriction (RFLP) a montré de façon indiscutable la réalité de l'homo- et de l'hémizygotie dans les cellules du rétinoblastome. Une situation tout à fait comparable existe dans le cas du néphroblastome avec aniridie (et souvent autres anomalies associées) et dans lequel à l'état constitutionnel peut exister une délétion de la bande i i pi3. Dans les cellules tumorales ellesmêmes, on a pu montrer soit par la cytogénétique, soit par les méthodes de la biologie moléculaire, qu'une hémizygotie ou une homozygotie pour le chromosome i I anormal pouvait exister [27]. Si les conséquences moléculaires de ces anomalies chromosomiques sont encore mal comprises, il semble que le phénomène lui-même soit plus fréquent que l'on ne croyait jusqu'à une date très récente, en particulier dans les tumeurs solides. L'hémiou l'homozygotie pour un chromosome i I a été observée en effet dans des néphroblastomes primitifs isolés sans malformations oculaires ou génitales associées [28], et il existe maintenant de nombreux exemples, étudiés à l'aide des techniques de la biologie moléculaire, montrant la perte d'un allèle ou d'un RFLP dans des tumeurs humaines variées.

\section{Conclusion}

La cytogénétique moderne a été profondément modifiée par de nouveaux apports technologiques: la cytométrie de flux permet d'ores et déjà de trier des chromosomes isolés et des banques d'ADN chromosomique humain sont actuellement disponibles. D'autre part, l'amélioration considérable de la sensibilité des techniques d'hybridation in situ par l'utilisation de sondes radioactives ou fluorescentes doit permettre de détecter et de localiser avec une précision de plus en plus 


\section{REFERENCES}

18. Shima E, Le Beau MM, McKeitham TW, et al. T-cell receptor $\alpha$-chain moves immediately downstream of $c$-myc in a chromosomal $8 ; 14$ translocation in a cell line from a human $\mathrm{T}$-cell leukaemia. Proc Natl Acad Sci USA (sous presse).

19. Groffen J, Stephenson JR, Heisterkamp N, de Klein A, Bartram K, Grosveld G. Philadelphia chromosomal breakpoints are clustered within a limited region bcr, on chromosome 22. Cell 1984 ; $36: 93: 9$

20. Shtivelman E, Lifshitz B, Gale RP, Canaani E. Fused transcripts of $a b l$ and bcr genes in chronic myelogenous leukaemia. Nature 1985; 315 : 550-4

21. Konopka JB, Watanabe S, Witte ON. An alteration of the human c-abl protein in $K_{5} 6_{2}$ leukaemia cells unmasks associated tyrosine kinase activity. Cell 1984; 37 : 1035-42.

22. Levan G, Levan A. Transitions of double minutes into homogeneously staining regions and C-bandless chromosomes in the SEWA tumor. In: Schimke RT, ed. Gene Amplification, Cold Spring Harbor Symp Quant Biol 1982 : 91-7.

23. Gcorge DL. Amplification of cellular protooncogenes in tumours and tumour cell lines. Cancer Surveys 1984; $3: 497-513$

24. Seeger RC, Brodeur GM, Sather H, et al. Association of multiple copies of the N-myc oncogene with rapid progression of neuroblastomas. New Engl 7 Med 1985; 313: i I 1 1-6.

25. Sparkes RS. Cytogenetics of retinoblastoma. Cancer Surveys 1984; 3: 479-96.

26. Murphree AL, Bencdict WF. Retinoblastoma : Clues to human oncogenesis. Science 1984; 223 : 1028-33.

27. Orkin SH. Wilms' tumour: Molecular evidence for the role of chromosome i1. Cancer Surve ys $1984 ; 3: 465-77$.

28. Kouf os A, Hansen MF, Copeland NG, Jenkins NA, Lampkin BC, Cavenee WK. Loss of heterozygosity in three embryonal tumours suggests a common pathogenetic mechanism. Nature 1985; 316 : 330-4.

29. Blanchard JM, Piechaczyk M, Dani C, et al. $c$-myc gene is transcribed at high rate in Go arrested fibroblasts and is post-transcriptionnally regulated in response to growth factors. Nature 1985; 3 1 7 : 443-5.

30. Piechaczyk M, Yang JQ Blanchard JM, Jeanteur P, Marcu K. Posttranscriptional mechanisms are responsible for accumulation of truncated $c$-myc RNAs in murine plasma cell tumors. Cell $1985 ; 42: 589-97$. fine des gènes à l'état de copie unique. L'utilisation conjuguée de ces deux techniques devrait aboutir à l'identification rapide des gènes altérés par les remaniements chromosomiques dans la plupart des cas individuels de tumeurs humaines, pour peu qu'une infrastructure appropriée soit mise en place.

D'un point de vue fondamental, même si l'on admet que des accidents chromosomiques non aléatoires conduisent à l'activation directe de gènes et en particulier de proto-oncogènes, on n'est pas encore en mesure de comprendre le mécanisme intime du phénomène, très certainement parce qu'on ne connait pas encore les fonctions normales de ces gènes. Il est clair que la diversité de leurs localisations subcellulaires (surface cellulaire, cytosol, noyau) et la variété de leurs activités, lorsqu'elles sont connues (protéines kinases, GTP-binding protéines, $A D N$-binding protéines) reflètent des fonctions et donc des régulations différentes pour chacun d'eux. Dans ce contexte, des résultats très récents remettent en cause notre connaissance de la régulation de l'expression du proto-oncogène $c$-myc. Contrairement à ce qu'on croyait jusqu'alors, le gène est transcrit à une vitesse quasi constante dans les cellules normales, même lorsqu'elles sont au repos [29]. Mais la stabilité (demi-vie) de l'ARNmessager varie très sensiblement selon l'état du cycle cellulaire, passant de quelques minutes dans des cellules quiescentes à quelques dizaines de minutes dans des cellules en cycle, pour atteindre quelques heures dans des cellules tumorales de plasmocytome de rat, l'équivalent murin du lymphome de Burkitt [3o]. Il reste à comprendre comment l'événement moléculaire issu de la translocation retentit sur la régulation post-transcriptionnelle du gène.

Finalement, l'accent mis ces derniers temps sur les oncogènes ne doit pas faire oublier que d'autres gènes, oncogènes ou non, sont certainement impliqués dans le développement des cancers. La cytogénétique doit jouer un rôle majeur qui permettra de connaître la localisation normale et pathologique de ces gènes

\section{Summary}

Chromosome abnormalities in malignant cells are clonal and nonrandom. Some are linked to specific types of malignant proliferation. Cytogenetic evidence of multiple gene amplification are homogeneously staining regions and double minute chromosomes. The use of molecular biology techniques as well as in situ hybridization and cell sorter methodology helped to show that certain genes such as proto-oncogenes are translocated. These chromosome rearrangements result in an abnormal gene regulation. In some solid tumors hemi- or homozygosity for specific chromosomes has been proved. Cytogenetics is a valuable tool to investigate molecular mechanisms involved in cancer.

\section{TIRES A PART}

R. Berger : Institut de recherches sur les maladies du sang, hôpital Saint-Louis, 2, place du Docteur Fournier, 75475 Paris Cedex Io. 